\title{
A Comparative Study on Dyeing Properties of Hemp and Cotton Fiber
}

\author{
Shekh Md. Mamun Kabir, PhD
}

Department of Wet Processing Engineering, Bangladesh University of Textiles, Bangladesh

\author{
Rezaul Karim, BSc \\ Khayrul Islam, BSc
}

Department of Textile Engineering, Sonargaon University, Bangladesh

Doi: 10.19044/esj.2017.v13n33p378 URL:http://dx.doi.org/10.19044/esj.2017.v13n33p378

\begin{abstract}
In this study, a mixed bi-functional reactive dye was applied to the cotton and hemp woven fabrics. Their dyeing and fastness properties were compared. From the results, it was observed that the cotton fabric exhibits better exhaustion and levelness than hemp woven fabrics. The build-up and fastness properties of the two woven fabrics appeared to be almost similar.
\end{abstract}

Keywords: Hemp, Amorphous, Dyeing properties, WAXD, Fastness properties

\section{Introduction}

Textile use for hemp (Cannabis sativa L.) was cultivated by mankind from the ancient time ( $\mathrm{Lu} \mathrm{X}$. et al., 1995). In the production of environmental clothes, focus on hemp fiber is great interest nowadays (Buschle D. et al., 1999. Dang V et al., 2006.Gumuskaya et al., 2007). As a textile fiber, hemp possesses a range of excellent properties such as, high tensile strength, low strain, high elasticity and breathability with quick drying. Hemp fiber also has got a good antimicrobial property, better moisture and thermal control, biodegradability and resistance to UV radiation (Wang $\mathrm{H}$ et al., 2003. Milosavljevic S et al., 2001). However, there are also some drawbacks of hemp fabrics such as higher stiffness and rough handle. The rigidity of cell wall is lignin which is located in the middle of lamellae and secondary wall. The amorphous and unoriented constituent are hemicellulose, which occupy spaces between the fibrils in both primary and secondary walls. Raw hemp fiber consists of about $67-78 \%$ cellulose, approximately $5.5-16.1 \%$ hemicelluloses, $3.7-8 \%$ lignin, $0.9-4.3 \%$ pectin and some fats and waxes (Buranov A et al., 2008. Chauhan A et al., 2013). Many 
researchers have been doing extensive study for improving the hand feel and physical properties of hemp and other fabrics (Sun Ji D et al., 2016. Li J et al., 2010).

Although the abundant of synthetic fiber, cotton (Gossypium hirsutum L.) is still most popular and versatile textile fiber in world market (Krifa M et al., 2016). It is used for a variety of textile applications such as apparel fabric, upholstery, carpets and curtains, etc (Schneider A. M. et al., 1991). Cotton fiber is smoother, stiffer and straighter than hemp fiber. Cotton can be dyed easily and the color of dyed cotton often remains fast to repeated washings and to prolonged wear as well (Mwaikambo L.Y. et al., 2002). Whereas hemp fiber is coarser than cotton; it is dark in color and difficult to bleach because of lignin. For the dyers, it's really challenging task to dye hemp fiber. Even though many studies have reported the dyeing of hemp fibers with direct dye. Also basic dyestuff provide bright shades when hemp is mordanted with antimony and tannin (Gordon Cook J. et al., 2003). There has been a limited researches reporting the dyeing properties of hemp fabric dyed with reactive dye.

In this study, a comparative analysis of the dyeing properties of hemp and cotton fiber dyed with bi-functional reactive dye through dye exhaustion method has been reported; the build-up properties, levelness and fastness properties have been discussed.

\section{Materials and Methods}

\section{Materials}

100\% Hemp (20's) and 100\% Cotton (20's) woven fabrics (plain weave) generously supplied by Beximco Textiles Ltd (Bangladesh).

Bi-functional reactive dye was not purified prior to use; the Sunfix Supra Red S3B 150\% (C.I. Reactive Red 195) (scheme 1) was obtained by the kind cooperation of Oh young corporation.

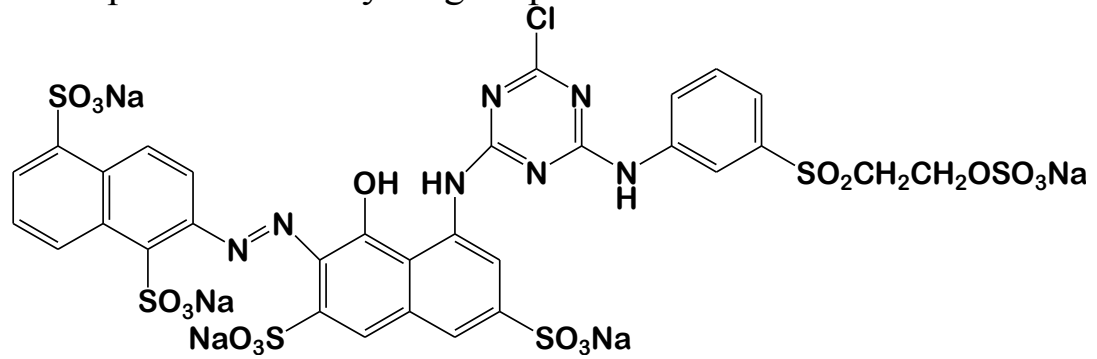

Figure 1- Bifunctional reactive dyes (C.I. Reactive Red 195).

A commercial grade salt $\left(\mathrm{Na}_{2} \mathrm{~S}_{2} \mathrm{O}_{4} \cdot 10 \mathrm{H}_{2} \mathrm{O}\right)$, alkali $(\mathrm{NaOH})$ and soaping agents was collected from Zhengzhou Sigma Chemical Company Ltd., China. All other reagents were of general purpose grade. 


\section{Wide-Angle X-ray Analysis}

The crystallinity and amorphousness of the hemp and cotton fibre were measured and analyzed by wide-angle $\mathrm{X}$-ray diffraction technique. The fibers were placed perpendicular or parallel to the direction of the X-ray beam in order to obtain equatorial and meridional X-ray diffraction data respectively. The experiment was carried out using Rich-Seifert (Model ISODebyeflex 2000) diffractometer. The WAXD pattern was obtained using $\mathrm{CuK}_{\alpha}$ radiation $\lambda=1.5418 \AA$ which was monochromatised with a curved graphite crystal monochromator and nickel filter. Intensities were measured within the range of scattering angles $(2 \theta)$ of $0-30^{\circ}$ in the equatorial and meridional directions. The total scattering data were obtained by the summation of both equatorial and meridional scattering data. Bragg diffraction peaks in the total scattering data were separated by a conventional curve fitting method using a commercial software.

\section{Dyeing}

A $50 \mathrm{ml}$ dye bath, suitable for a $2.0 \mathrm{~g}$ sample of hemp and cotton woven fabric (liquor ratio 1:20), containing reactive dye, Glauber's $\left(\mathrm{Na}_{2} \mathrm{~S}_{2} \mathrm{O}_{4} .10 \mathrm{H}_{2} \mathrm{O}\right)$ salt and soda ash $\left(\mathrm{Na}_{2} \mathrm{CO}_{3}\right)$ was prepared. Dyeing was performed for $60 \mathrm{~min}$ at $60^{\circ} \mathrm{C}$ in an Ahiba laboratory dyeing machine (Ahiba, Data color International, Switzerland). The dyeing method used is shown in Figure 1. After dyeing all the dyed samples were cleaned with soaping agent for $10 \mathrm{~min}$ at $95^{\circ} \mathrm{C}$ and rinsed with cold water and dried at room temperature.

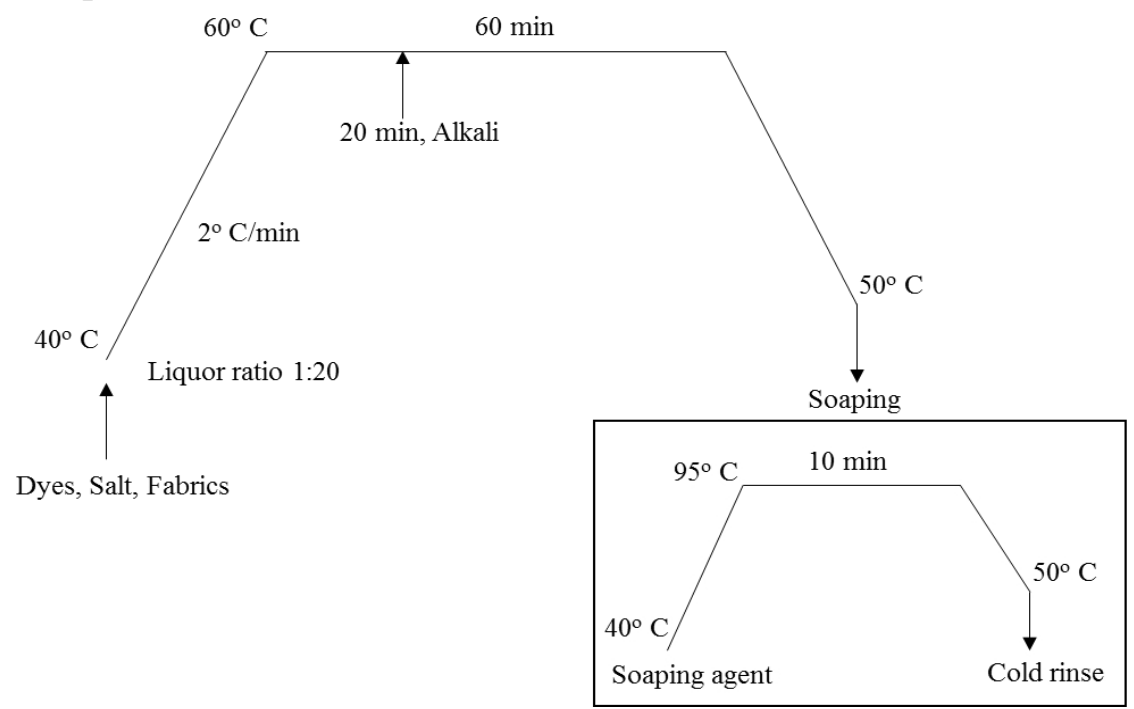

Figure 2- Dyeing profile for bi-functional reactive dye with hemp and cotton. 
The hemp and the cotton woven fabrics were dyed at various dye bath conditions (salt concentration, alkali concentration and dye concentration) in order to investigate their effects on the dyeing properties.

\section{Measurement of dye exhaustion and color strength}

After dyeing, a $3 \mathrm{ml}$ sample of the residual dye bath was removed to measure the absorbance value using a UV-vis spectrophotometer (Agilent, USA) and the percentage dye bath exhaustion $(\% \mathrm{E})$ was calculated by using Eqn (1):

$$
\text { Exhaustion }(\%)=\frac{A_{b}-A_{a}}{A_{b}} \quad \text { Eqn. } 1
$$

Where, $\quad A_{a}$ : Absorbance of the dye bath after dyeing $A_{b}:$ Absorbance of the dye bath before dyeing

The color strength $\left(f_{k}\right)$ and the color difference $(\Delta \mathrm{E})$ values of the dyed fabrics were measured using a spectrophotometer (X-Rite 8000 Series, standard light $\mathrm{D}_{65}, 10^{\circ}$ standard observer, specular component included; Xrite, USA) that was interfaced with a personal computer. Here, $f_{k}$ represents the color strength value as the sum of the weighted $\mathrm{K} / \mathrm{S}$ values in the visible region of the spectrum, as follows:

$$
f_{k}=\sum_{\lambda=400}^{700}\left(\frac{K}{S}\right)_{\lambda}\left(\bar{x}_{10, \lambda}+\bar{y}_{10, \lambda}+\bar{z}_{10, \lambda}\right)
$$

Where $\mathrm{x}_{10, \lambda}, \mathrm{y}_{10, \lambda}$, and $\mathrm{z}_{10, \lambda}$ are the color-matching functions for a $10^{\circ}$ standard observer at each wavelength (ISO 7724/1: 1984). The levelness of the dyed fabrics was measured using an instrumental method that was developed by Yang and Li (Yang and Li et al., 1993). The levelness of the dyed fabrics was excellent, so it's better to use the modified levelness parameter $\mathrm{L}$ the rating of which is very much similar to the grey scale rating "5" (for color change).

$$
\begin{gathered}
\mathrm{L}=1.2 \times(2.0-\ln \mathrm{U}) \quad(\mathrm{U} \geq 0.3114) \\
=5.0-1.2 \times \exp \left(\frac{7}{6}\right) \times \mathrm{U} \quad(\mathrm{U}<0.3114) \\
\text { Where } \mathrm{U}=\sum \mathrm{S}_{\mathrm{r}}(\lambda) \times \mathrm{V}(\lambda) \\
\mathrm{S}_{\mathrm{r}}(\lambda)=\text { Relative sample standard deviation of }(\mathrm{K} / \mathrm{S}) \lambda, \mathrm{V}(\lambda)=
\end{gathered}
$$
Spectral luminous function.

\section{Determination of color fastness properties}

The hemp and the cotton woven fabrics were dyed (1/1 standard depth), after-treated with soaping agent and heat-set $\left(170^{\circ} \mathrm{C}, 30 \mathrm{sec}\right)$ with a view to measuring the color fastness. The color fastness was assessed according to the corresponding international standards, including the fastness 
to washing (ISO 105-C06 A2S:2010), fastness to rubbing (ISO 105-X12) and fastness to perspiration (ISO 105-E04:2013). The changes in the shade and staining of the adjacent Multifibre (Multifibre DW, adjacent fabric, BS ISO 105-F10:1989 were assessed using grey scales.

\section{Results and Discussion}

\section{WAXD analysis of hemp and cotton fiber}

Figures 3 shows a fully developed crystalline structure with relatively enhanced crystalline orientation which is shown by lower azimuthal arcing of the equatorial and off-equatorial reflections. Both the figures illustrate an amorphous halo with several crystalline Bragg peaks. The information about the amorphous phase is contained in the weak amorphous halo underneath the intense crystalline peaks.

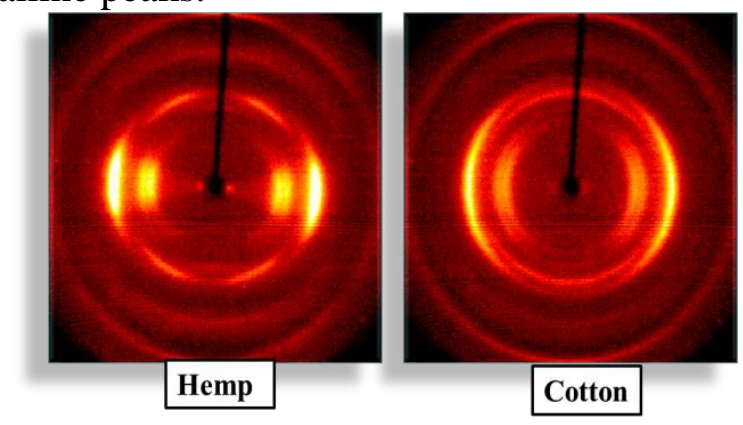

Figure 3. Fiber diagrams of hemp and cotton fibers.

The scattering from an unoriented amorphous phase is isotropic around the azimuth, whereas that from an oriented amorphous phase is intense along the equator, and weaker along the meridian, giving rise to an amorphous crescent. The positions in both the patterns are quite similar, indicating that the crystalline structures of both the fibers are almost similar. However, the peak width in the azimuthal direction for cotton is larger than that measured for hemp fiber, which demonstrates that the orientation of the crystalline phase of hemp is better than cotton fiber. Dyeing properties of fiber depend on the relative amount of amorphous phase and the loose chain packing especially in the intermediate phase between crystalline and amorphous phases. The structural parameters strongly affect the dye and solvent sorption of fibrous polymer. Figure 2 shows that the chain packing of hemp fiber is better than that of cotton fiber, which indicates poor dye ability of hemp fiber. Figure 3 depicts the total scattering data of cotton and hemp fiber measured at room temperature. The crystalline peaks contribute to the intensity of the selective diffracted radiation and the non-crystalline peaks appear as the intensity of the diffuse background. The Bragg peaks of both fibers are displayed by curve fitting method. In figure 3 , we found three 
Bragg peaks intensity at $14.98^{\circ}, 17.30^{\circ}, 23.25^{\circ}$ for hemp is which higher than that measured for the cotton fiber and this reveals that the crystallinity of cotton fiber is lower than that of hemp fiber.

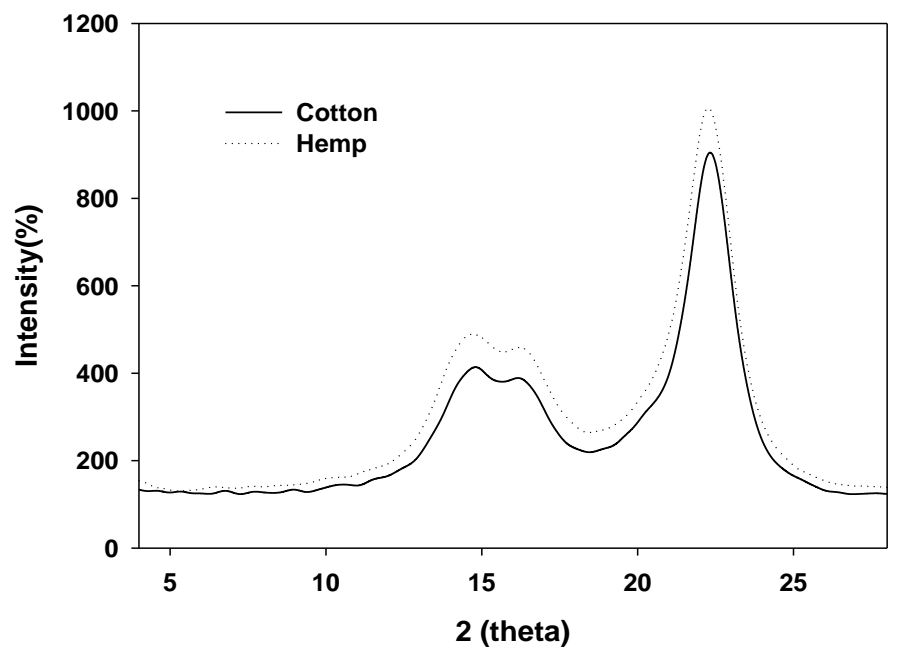

Figure 4. Wide angle X-ray diffraction pattern resolved into individual integral Bragg intensities and the amorphous background.

Most crystalline Bragg peaks of hemp and cotton fiber were located in the region of $2 \theta \leq 30^{\circ}$, their approximate crystallinities estimate based on the curve fitted data in Figure 4. The upper value of crystallinity of hemp fiber exhibits lower solvent process characteristics and dye ability than that of cotton fiber. The crystallinity (\%) of the cotton fiber is $75 \%$ and the hemp fiber is $89 \%$. On the other hand the crystal size of the cotton fiber is $5.5 \mathrm{~nm}$ and the hemp fiber is $2.8 \mathrm{~nm}$.

\section{Bi-functional reactive dye exhaustion on hemp and cotton fiber}

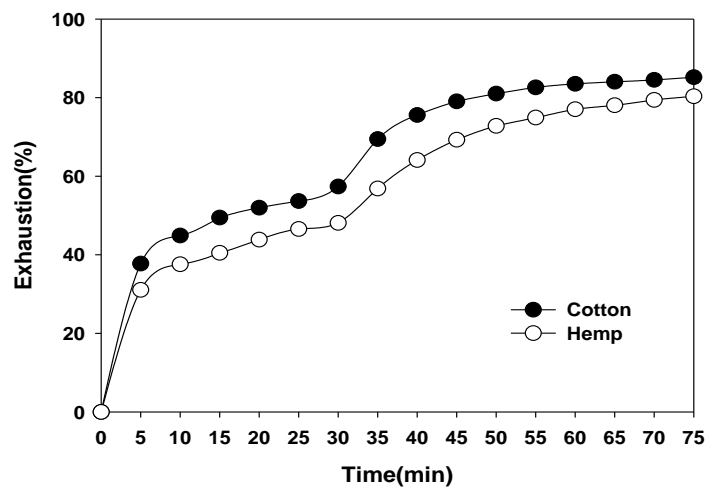

Figure 5. Dye exhaustion of bi-functional reactive dye on cotton and hemp fibers (dye conc. $1.0 \%$ owf, salt conc. $20 \mathrm{~g} / \mathrm{l}$ and material to liquor ratio 1:20). 
Figure 5 shows the exhaustion behavior of bi-functional reactive dye on cotton and hemp fibers during dyeing. The dyeing rate of the two fibers is not the same because of their molecular structures. Cotton fiber shows faster exhaustion behavior than hemp fiber, presumably due to the lower crystallinity and degree of orientation. The crystallites that lie preferentially parallel to the fiber axis and are separated by regions of lower order are strongly fiber specific. The figure 5 shows that cotton fiber gives $80 \%$ exhaustion because intermicellar spaces and regions of lower order of the fiber are accessible for dyestuff. However, highly oriented crystallites reduce the diffusion of dye into the fiber surface.

\section{Effect of salt concentration on dyeing properties}

Electrolytes are essential for dyeing with reactive dye. The addition of electrolyte directly affect the dye bath, which is called salt sensitivity. The addition of electrolyte increases the rate of strike of the dye. Glauber's salt $\left(\mathrm{Na}_{2} \mathrm{~S}_{2} \mathrm{O}_{4} .10 \mathrm{H}_{2} \mathrm{O}\right)$ and common salt $(\mathrm{NaCl})$ are mostly used as electrolytes. Reactive dyes for cellulosic fiber have negatively charged active groups and they are anionic. When cotton/hemp fiber is immersed in water, its surface due to the hydroxyl ions also become anionic, hence the dye particles and the cellulosic fiber tend to repel each other. So the level of substantivity is reduced. The addition of salt creates an electrical positive double layer which hides the negative electrostatic charge (Donnan Potential) of the cellulose surface. This allows the dye to make an approach to the fiber, allowing better interaction of Van der Waals forces as well; this improves the substantivity. If electrolyte is not spread out uniformly on cotton surface, dye distribution will not be even and patchy dyeing will become unavoidable. The diffusion coefficient of dye is therefore a function of the concentration of reactive dye and salt.

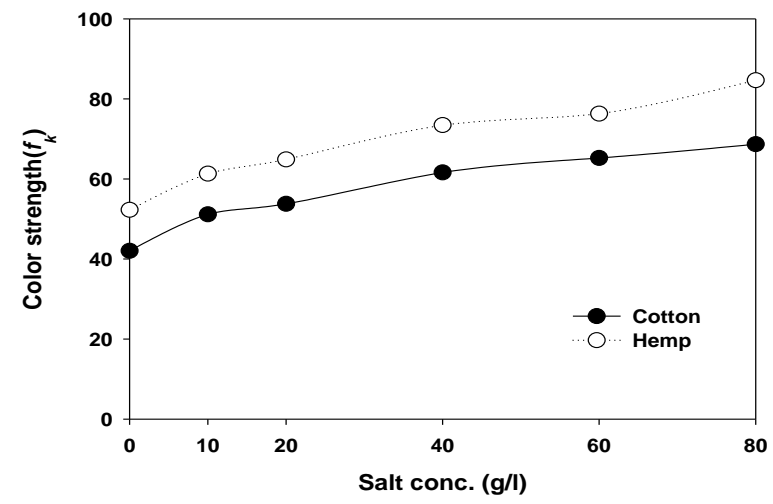

Figure 6. Effects of glauber's $\left(\mathrm{Na}_{2} \mathrm{~S}_{2} \mathrm{O}_{4} .10 \mathrm{H}_{2} \mathrm{O}\right)$ salt concentration on color strength $\left(f_{k}\right)$ of reactive dyeing (dye $1.0 \%$ owf, liquor ratio 1:20) with hemp and cotton fiber. 
Figure 6. shows that the color strength $\left(f_{\mathrm{k}}\right)$ of dyed fabrics increases with the increase in salt concentration. It is considered that increasing the electrolyte concentration reduces the electrical potential barrier, so dye diffusion increases and consequently the color strength increases both for hemp and cotton fibers. It is also evident from figure 4 that the color strength $\left(f_{\mathrm{k}}\right)$ of hemp is higher than that of cotton fiber upon the use of bi-functional reactive dye. It is clearly shows that when the salt concentration increases, the color strength increases slightly. The excellent color strength $\left(f_{\mathrm{k}}\right)$ could be ascribed to the lower crystal size; smaller crystal size means more surface area of the fiber and reduction in the distance between layers of cellulose which increases the capillary effect; therefore the accessibility to water and other chemicals to the fiber is increased. Since increasing the salt concentration decreases the electrical double layer, so bi-functional reactive dye penetrates faster into the hemp fiber than cotton fiber.

\section{Effect of Alkali Concentration on Dyeing Properties}

Figure 7. shows that the color strength $\left(f_{\mathrm{k}}\right)$ of dyed fabrics increases with the increase in alkali concentration. Alkali is originally used for dye fixation for reactive dyes.

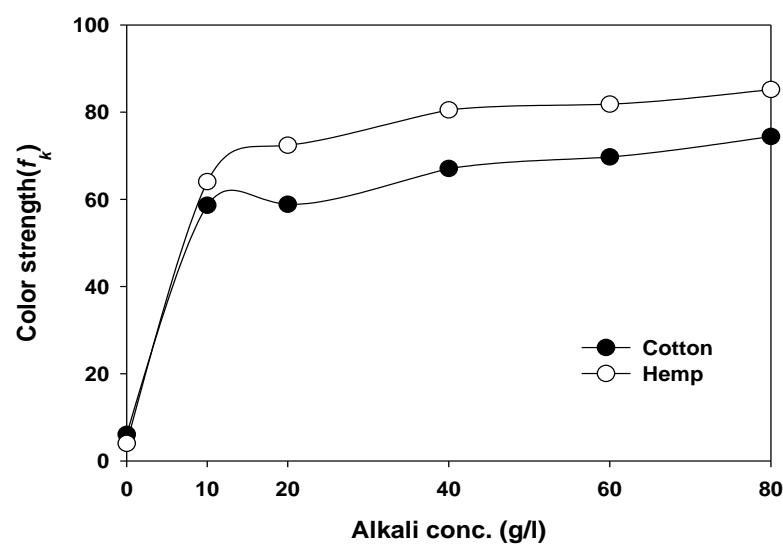

Figure 7. Effects of alkali $(\mathrm{NaOH})$ concentration on color strength $\left(f_{k}\right)$ of reactive dyeing (dye 1.0\% owf, liquor ratio 1:20) with hemp and cotton fiber.

It can be seen in figure 7 that the color strength $\left(f_{k}\right)$ of cotton and hemp fiber is in similar point at $10 \mathrm{~g} / \mathrm{l}$. When the concentration increased at $20 \mathrm{~g} / \mathrm{l}$, the color strength difference of two fiber increased, cotton fiber color strength is 60 and hemp fiber color strength is 75 . On the other hand the color strength $\left(f_{\mathrm{k}}\right)$ of the dye used to reach the saturation levels at $40 \mathrm{~g} / 1$, the data represents that color strength of the hemp fiber is 80 and the cotton fiber is 62. The mixed bifunctional reactive dye shows excellent exhaustion properties with addition of alkali. It can be explained that the larger surface 
area of hemp fiber enhances the more dye uptake by the influence of alkali than cotton fiber. Also alkali is used for removing the unfixed dyes on the fiber surface.

\section{Dyeing Behavior}

From the result obtained for the build-up properties upon the use of bi-functional reactive dye, it can be said that the dye ability of cotton and hemp is quite similar. When the dye concentration (\% owf) increases, color strength $\left(f_{k}\right)$ also increases for both the fibers.

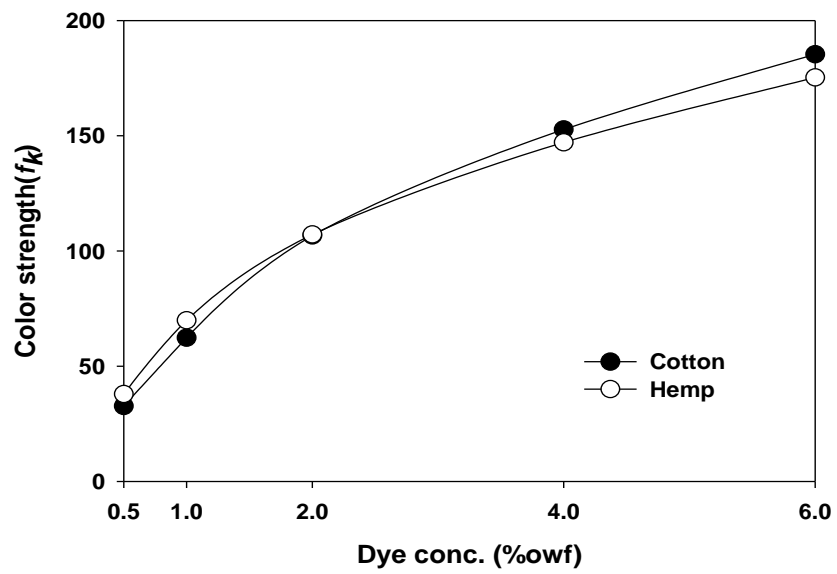

Figure 8. Build-up properties of reactive dye on cotton and hemp fibers (dye conc. 1.0\% owf, salt conc. $20 \mathrm{~g} / \mathrm{l}$ and material to liquor ratio 1:20).

At low concentration ( $0.5 \& 1 \%$ owf) dyeing of hemp fiber shows higher color strength than that of cotton fibers and the color strength of both the fibers superimposed at $2.0 \%$ owf dye concentration. When the dye concentration increases to $4 \%$ and $6 \%$ cotton fiber gives higher color strength than hemp fiber. At low concentration, hemp fiber absorbs more dyestuff than cotton because smaller crystal size enhances the dye up take; as the dye concentration increases, saturation point is observed in hemp fiber. Hence at higher dye concentration levels, hemp fiber shows lower color strength. On the other hand, the amorphousness of cotton fiber allows more dye uptake at higher concentration levels.

\section{Levelness of dyed fabrics}

The levelness of the dyed fabrics was assessed using an instrumental method that was developed by Yang and Li. In this study the levelness of dyed cotton and hemp fibers were found to be so excellent, which is obtain values 5. Figure 9 shows that when the dye concentration increases, the levelness parameter of both the dyed fabrics are decreased. Cotton fiber 
shows better levelness parameter than hemp fiber. It can be explained that, higher crystallinity prevents dye absorption into the fiber, so the levelness of dyed hemp fiber is lower than that of cotton fiber.

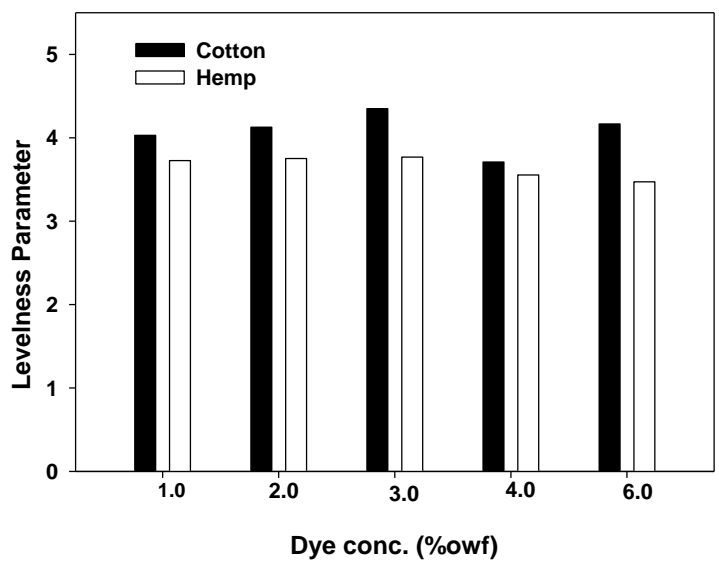

Figure 9. Levelness of reactive dyed cotton and hemp fabric (dye conc. 1.0\% owf, salt conc. $20 \mathrm{~g} / \mathrm{l}$ and material to liquor ratio 1:20).

\section{Colour fastness}

Appropriate color fastness for multiple end uses can be achieved through correct dye recipe selection and optimized finishing route design. Color fastness varies with the changes in fiber structure and properties. In order to study the influence of dyeing properties of cotton and hemp, we measured the color fastness to washing, rubbing and perspiration. Color fastness to wash is compared in table 1, the data represent a color change rating of "good" to "excellent" (with numerical grades 4-5 and 5).

Table 1. Wash fastness of the reactive dyes on cotton and hemp fiber

\begin{tabular}{cccccccc}
\hline Washing & \multicolumn{6}{c}{ Staining } & \multirow{2}{*}{ Change } \\
\cline { 2 - 7 } Fastness & Wool & Acrylic & Polyester & Nylon & Cotton & Acetate & \\
\hline Cotton & $4-5$ & 5 & $4-5$ & $4-5$ & $4-5$ & $4-5$ & 4 \\
Hemp & 5 & 5 & 5 & 5 & 5 & $4-5$ & $4-5$ \\
\hline
\end{tabular}

Wool and cotton were consistently found to be the most stained components of the multifibre for the reactive dyed cotton and hemp fabrics. The amorphousness of cotton fiber increases dye penetration into the fiber, so unfixed dyes move out from the fabric surface. However, the mixed bifunctional reactive dye shows excellent fastness results (rating of 5) for hemp fiber.

Color fastness to perspiration (table 2) of reactive dyed cotton and hemp fabrics were within the range of 4 to 5 . The results indicate that hemp fiber shows higher perspiration (both acidic and alkaline) fastness than cotton fiber. Moreover, in case of rubbing fastness, all of the dyed samples 
showed (in table 3) almost similar ratings. No significant difference in the color fastness property of reactive dyed cotton and hemp fabrics was detectable.

Table 2. Perspiration fastness of the reactive dyes on cotton and hemp fiber

\begin{tabular}{|c|c|c|c|c|c|c|c|}
\hline \multirow{2}{*}{\multicolumn{2}{|c|}{$\begin{array}{l}\text { Perspiration } \\
\text { Fastness }\end{array}$}} & \multicolumn{6}{|c|}{ Staining } \\
\hline & & Wool & Acrylic & Polyester & Nylon & Cotton & Acetate \\
\hline \multirow{2}{*}{ Alkali } & Cotton & $4-5$ & $4-5$ & 5 & 5 & 4 & $4-5$ \\
\hline & Hemp & 5 & 5 & 5 & 5 & $4-5$ & 5 \\
\hline \multirow[b]{2}{*}{ Acid } & Cotton & $4-5$ & $4-5$ & 5 & $4-5$ & $4-5$ & $4-5$ \\
\hline & Hemp & 5 & 5 & 5 & 5 & 5 & 5 \\
\hline
\end{tabular}

Table 3. Rubbing fastness of the reactive dyes on cotton and hemp fiber

\begin{tabular}{ccc}
\hline Rubbing & Wet & Dry \\
Fastness & & $4-5$ \\
\hline Cotton & $3-4$ & $4-5$ \\
Hemp & $3-4$ & \\
\hline
\end{tabular}

\section{Conclusion}

The dyeing properties of hemp fiber were found to be excellent in comparison with that of cotton fiber. The dye yield for the two cellulosic fibers upon using mixed bi-functional reactive dye confirms the interrelation between the molecular rearrangements with the dyeing properties. Cotton fiber exhibits higher exhaustion and better levelness properties than hemp fiber, presumably due to the lower crystallinity and degree of orientation. However, the build-up properties of both fibers are almost identical. Salt and alkali addition enhances the color strength of hemp fiber because of lower crystal size which enhances the surface area of the fiber. Fastness properties of reactive dyed cotton and hemp fabrics were found to be almost similar. Therefore, the results suggest that hemp fiber could be used as an alternative to cotton fiber as a cellulosic fiber.

\section{Acknowledgement}

This work was supported by the faculty research fund of Bangladesh University of Textiles in 2017.

\section{References:}

1. Lu X. and Clarke R.C. (1995). J. Int. Hemp Assoc. 2: 26.

2. Buschle Diller G., Fanter C., Loth F. (1999). Textile Research Journal 69244-251.

3. Dang V., Nguyen K.L. (2006). Bioresource Technology 97:13531359.

4. Gumuskaya E., Usta M., Balaban M. (2007). Bioresource Technology 98: 491-497. 
5. Kostic M., Pejic B., Skundric P. (2008). Bioresource Technology 99: 94-99.

6. Wang H., Postle R., Kessler R., Kessler W. (2003). Textile Research Journal 73: 664-669.

7. Milosavljevic S., Tadic T. (2001).The Indian Textile Journal 112:3136.

8. Mussig J., Martens R., Haring H. (1998). Textile Asia 29: 39-50.

9. Chauhan A., Chauhan P. (2013). J Chem Eng Process Technol, http://dx.doi.org/10.4172/2157-7048.S6-003.

10. Sun Ji D. and Jin Lee J. (2016). Fibers and Polymers, 17:143-150.

11. Li J., Feng J., Zhang $\mathrm{H}$ and Zhang J. (2010). Fibres and Textile in Eastern Europe, 18: 81.

12. Berkley E. (1948). Textile Research Journal, 2: 71-88.

13. Krifa M., Stevens S. S. (2016). Agricultural Sciences, 7747-758.

14. Schneider A.M. and Holcombe B.V. (1991). Textile Research Journal, 61: 488-494.

15. Mwaikambo L.Y, Ansell M.P. (2002). Journal of Applied Polymer Science, 84:2222-2234.

16. Xiang-gang Y., Jun-Kai H., Ruo-ying Z. (2003). Journal of Tianjin Institute of Textile Science and Technology.

17. Gordon Cook J. (2003), Handbook of Textile Fibres, (Woodhead Publishing Limited) 17.

18. Koh J. (2005), Indian Journal of Fibre \& Textile Research 3088-93.

19. Yang Y. and Li S. (1993). Text. Chem. Colorist 25:75.

20. Mamun Kabir S. M., Koh J. (2017), Coloration Technology 133: 2092174. 